\title{
SOME EXACT ALGEBRAIC EXPRESSIONS FOR THE TAILS OF TASOEV CONTINUED FRACTIONS \\ TAKAO KOMATSU
}

\author{
(Received 23 February 2011; accepted 16 January 2012) \\ Communicated by I. E. Shparlinski \\ In memory of Alfred J. van der Poorten
}

\begin{abstract}
Denote the $n$th convergent of the continued fraction $\alpha=\left[a_{0} ; a_{1}, a_{2}, \ldots\right]$ by $p_{n} / q_{n}=\left[a_{0} ; a_{1}, \ldots, a_{n}\right]$. In this paper we give exact formulae for the quantities $D_{n}:=q_{n} \alpha-p_{n}$ in several typical types of Tasoev continued fractions. A simple example of the type of Tasoev continued fraction considered is $\alpha=\left[0 ; u a, u a^{2}, u a^{3}, \ldots\right]$.

2010 Mathematics subject classification: primary 11A55; secondary 05A19, $11 \mathrm{~J} 70$.

Keywords and phrases: Tasoev continued fractions, Hurwitz continued fractions, continued fractions, Diophantine approximations.
\end{abstract}

\section{Introduction}

Denote by $\alpha=\left[a_{0} ; a_{1}, a_{2}, \ldots\right]$ the regular (or simple) continued fraction expansion of a real number $\alpha$, where

$$
\begin{gathered}
\alpha=a_{0}+1 / \alpha_{1}, \quad a_{0}=\lfloor\alpha\rfloor, \\
\alpha_{n}=a_{n}+1 / \alpha_{n+1}, \quad a_{n}=\left\lfloor\alpha_{n}\right\rfloor
\end{gathered}
$$

with $n \geq 1$. Let $p_{n} / q_{n}=\left[a_{0} ; a_{1}, \ldots, a_{n}\right]$ denote the $n$th convergent associated with $\alpha$. It is well known that $p_{n} / q_{n}$ (where $n=0,1,2, \ldots$ ) are good approximations of $\alpha$ in the sense of the inequality

$$
\left|q_{n} \alpha-p_{n}\right|<\frac{1}{a_{n+1} q_{n}} .
$$

More precisely,

$$
D_{n}:=q_{n} \alpha-p_{n}=\frac{(-1)^{n}}{\alpha_{n+1} q_{n}+q_{n-1}} \quad \forall n \geq 0
$$

(see, for instance, [1, Lemma 5.4]).

This work was supported in part by Grant-in-Aid for Scientific Research (C) (No. 22540005), the Japan Society for the Promotion of Science.

(C) 2012 Australian Mathematical Publishing Association Inc. 1446-7887/2012 \$16.00 
Quasi-periodic continued fractions have the form

$$
\begin{aligned}
\alpha & =\left[a_{0} ; a_{1}, \ldots, a_{n}, \overline{Q_{1}(k), \ldots, Q_{p}(k)}\right]_{k=1}^{\infty} \\
& =\left[a_{0} ; a_{1}, \ldots, a_{n}, Q_{1}(1), \ldots, Q_{p}(1), Q_{1}(2), \ldots, Q_{p}(2), Q_{1}(3), \ldots\right],
\end{aligned}
$$

where $a_{0}$ is an integer, $a_{1}, \ldots, a_{n}$ are positive integers, $Q_{1}, \ldots, Q_{p}$ are functions that take positive integral values for all $k=1,2, \ldots$, at least one of which is not constant. If each $Q_{j}$ has polynomial form (or is constant), the expansion of $\alpha$ is called a Hurwitz continued fraction (see, for instance, [16]). Well-known examples are

$$
\begin{gathered}
e=[2 ; 1,2,1,1,4,1,1,6,1, \ldots]=[2 ; \overline{1,2 k, 1}]_{k=1}^{\infty}, \\
\tanh 1=\frac{e^{2}-1}{e^{2}+1}=[0 ; 1,3,5,7, \ldots]=[0 ; \overline{2 k-1}]_{k=1}^{\infty}, \\
\tan 1=[1 ; 1,1,1,3,1,1,5,1, \ldots]=[1 ; \overline{2 k-1,1}]_{k=1}^{\infty} .
\end{gathered}
$$

If each $Q_{j}$ has exponential form (or is constant), the expansion of $\alpha$ is called a Tasoev continued fraction [3-7, 17]. In [4], the author found some more general Tasoev continued fractions:

$$
\begin{gathered}
{\left[0 ; \overline{u a^{k}}\right]_{k=1}^{\infty}=\frac{\sum_{n=0}^{\infty} u^{-2 n-1} a^{-(n+1)^{2}} \prod_{i=1}^{n}\left(a^{2 i}-1\right)^{-1}}{\sum_{n=0}^{\infty} u^{-2 n} a^{-n^{2}} \prod_{i=1}^{n}\left(a^{2 i}-1\right)^{-1}},} \\
{\left[0 ; u a-1, \overline{1, u a^{k+1}-2}\right]_{k=1}^{\infty}=\frac{\sum_{n=0}^{\infty}(-1)^{n} u^{-2 n-1} a^{-(n+1)^{2}} \prod_{i=1}^{n}\left(a^{2 i}-1\right)^{-1}}{\sum_{n=0}^{\infty}(-1)^{n} u^{-2 n} a^{-n^{2}} \prod_{i=1}^{n}\left(a^{2 i}-1\right)^{-1}}} \\
{\left[0 ; \overline{u a^{k}, v a^{k}}\right]_{k=1}^{\infty}=\frac{\sum_{n=0}^{\infty} u^{-n-1} v^{-n} a^{-(n+1)(n+2) / 2} \prod_{i=1}^{n}\left(a^{i}-1\right)^{-1}}{\sum_{n=0}^{\infty} u^{-n} v^{-n} a^{-n(n+1) / 2} \prod_{i=1}^{n}\left(a^{i}-1\right)^{-1}}}
\end{gathered}
$$

and

$$
\begin{aligned}
{[0 ; u a} & \left.-1,1, v a-2, \overline{1, u a^{k+1}-2,1, v a^{k+1}-2}\right]_{k=1}^{\infty} \\
& =\frac{\sum_{n=0}^{\infty}(-1)^{n} u^{-n-1} v^{-n} a^{-(n+1)(n+2) / 2} \prod_{i=1}^{n}\left(a^{i}-1\right)^{-1}}{\sum_{n=0}^{\infty}(-1)^{n} u^{-n} v^{-n} a^{-n(n+1) / 2} \prod_{i=1}^{n}\left(a^{i}-1\right)^{-1}} .
\end{aligned}
$$

One might say that Tasoev continued fractions are geometric and Hurwitz continued fractions are arithmetic [5]. Roughly speaking, $u a^{k}$ in a Tasoev continued fraction corresponds to $u(a+b k)$ in a Hurwitz continued fraction, where $k=1,2, \ldots$ The Tasoev continued fractions corresponding to $e$-type Hurwitz continued fractions were also derived in [6]:

$$
\begin{aligned}
& {\left[0 ; \overline{u a^{k}-1,1, v-1}\right]_{k=1}^{\infty}} \\
& \quad=\frac{\sum_{n=0}^{\infty} u^{-2 n-1} v^{-2 n} a^{-(n+1)^{2}} \prod_{i=1}^{n}\left(a^{2 i}-1\right)^{-1}}{\sum_{n=0}^{\infty}\left((u v)^{-2 n} a^{-n^{2}}-(u v)^{-2 n-1} a^{-(n+1)^{2}}\right) \prod_{i=1}^{n}\left(a^{2 i}-1\right)^{-1}}
\end{aligned}
$$


and

$$
\begin{aligned}
& {\left[0 ; \overline{v-1,1, u a^{k}-1}\right]_{k=1}^{\infty}} \\
& \quad=\frac{\sum_{n=0}^{\infty}\left(u^{-2 n} v^{-2 n-1} a^{-n^{2}}+u^{-2 n-1} v^{-2 n-2} a^{-(n+1)^{2}}\right) \prod_{i=1}^{n}\left(a^{2 i}-1\right)^{-1}}{\sum_{n=0}^{\infty}(u v)^{-2 n} a^{-n^{2}} \prod_{i=1}^{n}\left(a^{2 i}-1\right)^{-1}} .
\end{aligned}
$$

The different types of Tasoev continued fractions with period 3 shown in [5] are

$$
\begin{gathered}
{\left[0 ; \overline{u a^{2 k-1}-1,1, v a^{2 k}-1}\right]_{k=1}^{\infty}=\frac{\sum_{n=0}^{\infty} u^{-n-1} v^{-n} a^{-(n+1)^{2}} \prod_{i=1}^{n}\left(a^{2 i}-(-1)^{i}\right)^{-1}}{\sum_{n=0}^{\infty}(-1)^{n} u^{-n} v^{-n} a^{-n^{2}} \prod_{i=1}^{n}\left(a^{2 i}-(-1)^{i}\right)^{-1}},} \\
{\left[0 ; u a, \overline{v a^{2 k}-1,1, u a^{2 k+1}-1}\right]_{k=1}^{\infty}} \\
=\frac{\sum_{n=0}^{\infty}(-1)^{n} u^{-n-1} v^{-n} a^{-(n+1)^{2}} \prod_{i=1}^{n}\left(a^{2 i}-(-1)^{i}\right)^{-1}}{\sum_{n=0}^{\infty} u^{-n} v^{-n} a^{-n^{2}} \prod_{i=1}^{n}\left(a^{2 i}-(-1)^{i}\right)^{-1}}
\end{gathered}
$$

and so on.

More Tasoev-type continued fractions have been found by Mc Laughlin [14] and by the author $[9,10]$.

Some Diophantine properties of Tasoev continued fractions are characterized as properties of Ramanujan-type $q$-continued fractions [13]. The purpose of this paper is to examine Diophantine properties of Tasoev continued fractions. In particular, the error values $D_{n}$ for such continued fractions are explicitly given in Sections 2 and 3. A representative proof for one of these results is given in Section 4. Sections 5 and 6 consider Diophantine approximations of Hurwitz continued fractions. The error value $D_{n}$ can be expressed in terms of integrals in the cases of some typical Hurwitz continued fractions [2, 11, 12, 15], and these expressions are given in Section 7.

\section{Main results}

Let $p_{n} / q_{n}$ be the $n$th convergent of the Tasoev continued fraction

$$
\left[0 ; \overline{u a^{2 k-1}, v a^{2 k}}\right]_{k=1}^{\infty}=\frac{\sum_{n=0}^{\infty} u^{-n-1} v^{-n} a^{-(n+1)^{2}} \prod_{i=1}^{n}\left(a^{2 i}-1\right)^{-1}}{\sum_{n=0}^{\infty}(u v)^{-n} a^{-n^{2}} \prod_{i=1}^{n}\left(a^{2 i}-1\right)^{-1}} .
$$

Theorem 1. For all $k \geq 1$,

$$
\begin{gathered}
D_{2 k-1}=-\frac{\sum_{n=0}^{\infty}(u v)^{-n-k} a^{-n^{2}-4 k n-k(2 k+1)} \prod_{i=1}^{n}\left(a^{2 i}-1\right)^{-1}}{\sum_{n=0}^{\infty}(u v)^{-n} a^{-n^{2}} \prod_{i=1}^{n}\left(a^{2 i}-1\right)^{-1}}, \\
D_{2 k}=\frac{\sum_{n=0}^{\infty} u^{-n-k-1} v^{-n-k} a^{-n^{2}-(4 k+2) n-(k+1)(2 k+1)} \prod_{i=1}^{n}\left(a^{2 i}-1\right)^{-1}}{\sum_{n=0}^{\infty}(u v)^{-n} a^{-n^{2}} \prod_{i=1}^{n}\left(a^{2 i}-1\right)^{-1}} .
\end{gathered}
$$


REMARK 2. If $u=v$, then these identities become those of (1). If $u$ is replaced by $u a$ and $a$ is replaced by $a^{1 / 2}$, then they become those of (3).

Let $p_{n} / q_{n}$ be the $n$th convergent of the Tasoev continued fraction

$$
\begin{aligned}
{[0 ; u a} & \left.-1, \overline{1, v a^{2 k}-2,1, u a^{2 k+1}-2}\right]_{k=1}^{\infty} \\
& =\frac{\sum_{n=0}^{\infty}(-1)^{n} u^{-n-1} v^{-n} a^{-(n+1)^{2}} \prod_{i=1}^{n}\left(a^{2 i}-1\right)^{-1}}{\sum_{n=0}^{\infty}(-1)^{n}(u v)^{-n} a^{-n^{2}} \prod_{i=1}^{n}\left(a^{2 i}-1\right)^{-1}} .
\end{aligned}
$$

Theorem 3. For all $k \geq 1$,

$$
\begin{gathered}
D_{4 k-2}=\frac{\sum_{n=0}^{\infty}(-1)^{n}(u v)^{-n-k} a^{-n^{2}-4 k n-k(2 k+1)} \prod_{i=1}^{n}\left(a^{2 i}-1\right)^{-1}}{\sum_{n=0}^{\infty}(-1)^{n}(u v)^{-n} a^{-n^{2}} \prod_{i=1}^{n}\left(a^{2 i}-1\right)^{-1}} \\
D_{4 k}=\frac{\sum_{n=0}^{\infty}(-1)^{n} u^{-n-k-1} v^{-n-k} a^{-n^{2}-(4 k+2) n-(k+1)(2 k+1)} \prod_{i=1}^{n}\left(a^{2 i}-1\right)^{-1}}{\sum_{n=0}^{\infty}(-1)^{n}(u v)^{-n} a^{-n^{2}} \prod_{i=1}^{n}\left(a^{2 i}-1\right)^{-1}}
\end{gathered}
$$

Remark 4. For odd $k$, the recurrence relation $D_{k}=D_{k+1}-D_{k-1}$ gives a formula for $D_{k}$.

If $u=v$, then these identities become those of (2). If $u$ is replaced by $u a$ and $a$ is replaced by $a^{1 / 2}$, then these become those of (4).

\section{3. $e$-type Tasoev continued fractions}

Let $p_{n} / q_{n}$ be the $n$th convergent of the Tasoev continued fraction (5):

$$
\left[0 ; \overline{u a^{k}-1,1, v-1}\right]_{k=1}^{\infty}=\frac{\sum_{n=0}^{\infty} u^{-2 n-1} v^{-2 n} a^{-(n+1)^{2}} \prod_{i=1}^{n}\left(a^{2 i}-1\right)^{-1}}{\sum_{n=0}^{\infty}\left((u v)^{-2 n} a^{-n^{2}}-(u v)^{-2 n-1} a^{-(n+1)^{2}}\right) \prod_{i=1}^{n}\left(a^{2 i}-1\right)^{-1}} .
$$

Theorem 5. For all $k \geq 1$,

$$
\begin{gathered}
D_{3 k-1}=(-1)^{k} \sum_{n=0}^{\infty}\left((u v)^{-2 n-k-1} a^{-n^{2}-2(k+1) n-(k+1)(k+2) / 2}\right. \\
\left.-(u v)^{-2 n-k} a^{-n^{2}-2 k n-k(k+1) / 2}\right) \prod_{i=1}^{n}\left(a^{2 i}-1\right)^{-1} \\
\times\left(\sum_{n=0}^{\infty}\left((u v)^{-2 n} a^{-n^{2}}-(u v)^{-2 n-1} a^{-(n+1)^{2}}\right) \prod_{i=1}^{n}\left(a^{2 i}-1\right)^{-1}\right)^{-1}, \\
D_{3 k}=\frac{(-1)^{k} \sum_{n=0}^{\infty} u^{-2 n-k-1} v^{-2 n-k} a^{-n^{2}-2(k+1) n-(k+1)(k+2) / 2} \prod_{i=1}^{n}\left(a^{2 i}-1\right)^{-1}}{\sum_{n=0}^{\infty}\left((u v)^{-2 n} a^{-n^{2}}-(u v)^{-2 n-1} a^{-(n+1)^{2}}\right) \prod_{i=1}^{n}\left(a^{2 i}-1\right)^{-1}} .
\end{gathered}
$$


ReMark 6. The recurrence relation $D_{3 k-2}=D_{3 k-1}-D_{3 k-3}$ gives a formula for $D_{3 k-2}$.

Let $p_{n} / q_{n}$ be the $n$th convergent of the Tasoev continued fraction (6):

$$
\begin{aligned}
& {\left[0 ; \overline{v-1,1, u a^{k}-1}\right]_{k=1}^{\infty} \text {. }} \\
& \quad=\frac{\sum_{n=0}^{\infty}\left(u^{-2 n} v^{-2 n-1} a^{-n^{2}}+u^{-2 n-1} v^{-2 n-2} a^{-(n+1)^{2}}\right) \prod_{i=1}^{n}\left(a^{2 i}-1\right)^{-1}}{\sum_{n=0}^{\infty}(u v)^{-2 n} a^{-n^{2}} \prod_{i=1}^{n}\left(a^{2 i}-1\right)^{-1}} .
\end{aligned}
$$

Theorem 7. For all $k \geq 1$,

$$
\begin{aligned}
& D_{3 k-1}= \frac{(-1)^{k-1} \sum_{n=0}^{\infty}(u v)^{-2 n-k} a^{-n^{2}-2 k n-k(k+1) / 2} \prod_{i=1}^{n}\left(a^{2 i}-1\right)^{-1}}{\sum_{n=0}^{\infty}(u v)^{-2 n} a^{-n^{2}} \prod_{i=1}^{n}\left(a^{2 i}-1\right)^{-1}}, \\
& D_{3 k}=(-1)^{k} \sum_{n=0}^{\infty}\left(u^{-2 n-k} v^{-2 n-k-1} a^{-n^{2}-2 k n-k(k+1) / 2}\right. \\
&\left.+u^{-2 n-k-1} v^{-2 n-k-2} a^{-n^{2}-2(k+1) n-(k+1)(k+2) / 2}\right) \prod_{i=1}^{n}\left(a^{2 i}-1\right)^{-1} \\
& \\
& \quad \times\left(\sum_{n=0}^{\infty}(u v)^{-2 n} a^{-n^{2}} \prod_{i=1}^{n}\left(a^{2 i}-1\right)^{-1}\right)^{-1} .
\end{aligned}
$$

Remark 8. The recurrence relation $D_{3 k-2}=D_{3 k-1}-D_{3 k-3}$ gives a formula for $D_{3 k-2}$.

Let $p_{n} / q_{n}$ be the $n$th convergent of the Tasoev continued fraction (7):

$$
\left[0 ; \overline{u a^{2 k-1}-1,1, v a^{2 k}-1}\right]_{k=1}^{\infty}=\frac{\sum_{n=0}^{\infty} u^{-n-1} v^{-n} a^{-(n+1)^{2}} \prod_{i=1}^{n}\left(a^{2 i}-(-1)^{i}\right)^{-1}}{\sum_{n=0}^{\infty}(-1)^{n} u^{-n} v^{-n} a^{-n^{2}} \prod_{i=1}^{n}\left(a^{2 i}-(-1)^{i}\right)^{-1}} .
$$

Theorem 9. For all $k \geq 1$,

$$
\begin{aligned}
& D_{3 k-1}=\frac{(-1)^{k-1} \sum_{n=0}^{\infty}(-1)^{n}(u v)^{-n-k} a^{-n^{2}-4 k n-k(2 k+1)} \prod_{i=1}^{n}\left(a^{2 i}-(-1)^{i}\right)^{-1}}{\sum_{n=0}^{\infty}(-1)^{n} u^{-n} v^{-n} a^{-n^{2}} \prod_{i=1}^{n}\left(a^{2 i}-(-1)^{i}\right)^{-1}}, \\
& D_{3 k}=\frac{(-1)^{k} \sum_{n=0}^{\infty} u^{-n-k-1} v^{-n-k} a^{-n^{2}-(4 k+2) n-(k+1)(2 k+1)} \prod_{i=1}^{n}\left(a^{2 i}-(-1)^{i}\right)^{-1}}{\sum_{n=0}^{\infty}(-1)^{n} u^{-n} v^{-n} a^{-n^{2}} \prod_{i=1}^{n}\left(a^{2 i}-(-1)^{i}\right)^{-1}} .
\end{aligned}
$$

REMARK 10. The recurrence relation $D_{3 k-2}=D_{3 k}-D_{3 k-1}$ gives a formula for $D_{3 k-2}$.

Let $p_{n} / q_{n}$ be the $n$th convergent of the Tasoev continued fraction (8):

$$
\left[0 ; u a, \overline{v a^{2 k}-1,1, u a^{2 k+1}-1}\right]_{k=1}^{\infty}=\frac{\sum_{n=0}^{\infty}(-1)^{n} u^{-n-1} v^{-n} a^{-(n+1)^{2}} \prod_{i=1}^{n}\left(a^{2 i}-(-1)^{i}\right)^{-1}}{\sum_{n=0}^{\infty} u^{-n} v^{-n} a^{-n^{2}} \prod_{i=1}^{n}\left(a^{2 i}-(-1)^{i}\right)^{-1}} .
$$


THEOREM 11. For all $k \geq 1$,

$$
\begin{gathered}
D_{3 k}=\frac{(-1)^{k} \sum_{n=0}^{\infty}(-1)^{n} u^{-n-k-1} v^{-n-k} a^{-n^{2}-(4 k+2) n-(k+1)(2 k+1)} \prod_{i=1}^{n}\left(a^{2 i}-(-1)^{i}\right)^{-1}}{\sum_{n=0}^{\infty} u^{-n} v^{-n} a^{-n^{2}} \prod_{i=1}^{n}\left(a^{2 i}-(-1)^{i}\right)^{-1}}, \\
D_{3 k-2}=\frac{(-1)^{k} \sum_{n=0}^{\infty}(u v)^{-n-k} a^{-n^{2}-4 k n-k(2 k+1)} \prod_{i=1}^{n}\left(a^{2 i}-(-1)^{i}\right)^{-1}}{\sum_{n=0}^{\infty} u^{-n} v^{-n} a^{-n^{2}} \prod_{i=1}^{n}\left(a^{2 i}-(-1)^{i}\right)^{-1}}
\end{gathered}
$$

REMARK 12. The recurrence relation $D_{3 k-1}=D_{3 k}-D_{3 k-2}$ gives a formula for $D_{3 k-1}$. Lemma 13. For all $n \geq 0$,

$$
\begin{gathered}
p_{3 n}=\sum_{v=0}^{n-1}(-1)^{n-v-1} u^{v} v^{v+1} a^{(v+2)(2 v+1)} \prod_{i=1}^{n-v-1} \frac{a^{2(2 v+i+1)}+(-1)^{i}}{a^{2 i}-(-1)^{i}}, \\
p_{3 n+1}=\sum_{v=0}^{n}(-1)^{n-v}(u v)^{v} a^{v(2 v+3)} \prod_{i=1}^{n-v} \frac{a^{2(2 v+i)}-(-1)^{i}}{a^{2 i}-(-1)^{i}}, \\
q_{3 n}=\sum_{v=0}^{n}(u v)^{v} a^{v(2 v+1)} \prod_{i=1}^{n-v} \frac{a^{2(2 v+i)}-(-1)^{i}}{a^{2 i}-(-1)^{i}}, \\
q_{3 n+1}=\sum_{v=0}^{n} u^{v+1} v^{v} a^{(v+1)(2 v+1)} \prod_{i=1}^{n-v} \frac{a^{2(2 v+i+1)}+(-1)^{i}}{a^{2 i}-(-1)^{i}} .
\end{gathered}
$$

\section{Proof of Theorem 1}

We prove Theorem 1 by induction. The other theorems can be proven similarly. Let

$$
\alpha=\left[0 ; \overline{u a^{2 k-1}, v a^{2 k}}\right]_{k=1}^{\infty}=\frac{\sum_{n=0}^{\infty} u^{-n-1} v^{-n} a^{-(n+1)^{2}} \prod_{i=1}^{n}\left(a^{2 i}-1\right)^{-1}}{\sum_{n=0}^{\infty}(u v)^{-n} a^{-n^{2}} \prod_{i=1}^{n}\left(a^{2 i}-1\right)^{-1}} .
$$

The identities hold for $D_{0}$ and $D_{1}$ because

$$
\begin{aligned}
D_{0} & =q_{0} \alpha-p_{0}=\alpha \\
& =\frac{\sum_{n=0}^{\infty} u^{-n-1} v^{-n} a^{-(n+1)^{2}} \prod_{i=1}^{n}\left(a^{2 i}-1\right)^{-1}}{\sum_{n=0}^{\infty}(u v)^{-n} a^{-n^{2}} \prod_{i=1}^{n}\left(a^{2 i}-1\right)^{-1}}
\end{aligned}
$$

and

$$
\begin{aligned}
D_{1} & =q_{1} \alpha-p_{1}=u a \alpha-1 \\
& =\frac{\left(u a \sum_{n=0}^{\infty} u^{-n-1} v^{-n} a^{-(n+1)^{2}}-\sum_{n=0}^{\infty}(u v)^{-n} a^{-(n+1)^{2}}\right) \prod_{i=1}^{n}\left(a^{2 i}-1\right)^{-1}}{\sum_{n=0}^{\infty}(u v)^{-n} a^{-n^{2}} \prod_{i=1}^{n}\left(a^{2 i}-1\right)^{-1}} \\
& =\frac{\sum_{n=0}^{\infty}(u v)^{-n} a^{-n^{2}-2 n}\left(1-a^{2 n}\right) \prod_{i=1}^{n}\left(a^{2 i}-1\right)^{-1}}{\sum_{n=0}^{\infty}(u v)^{-n} a^{-n^{2}} \prod_{i=1}^{n}\left(a^{2 i}-1\right)^{-1}}
\end{aligned}
$$




$$
\begin{aligned}
& =-\frac{\sum_{n=1}^{\infty}(u v)^{-n} a^{-n^{2}-2 n} \prod_{i=1}^{n-1}\left(a^{2 i}-1\right)^{-1}}{\sum_{n=0}^{\infty}(u v)^{-n} a^{-n^{2}} \prod_{i=1}^{n}\left(a^{2 i}-1\right)^{-1}} \\
& =-\frac{\sum_{n=0}^{\infty}(u v)^{-n-1} a^{-n^{2}-4 n-3} \prod_{i=1}^{n}\left(a^{2 i}-1\right)^{-1}}{\sum_{n=0}^{\infty}(u v)^{-n} a^{-n^{2}} \prod_{i=1}^{n}\left(a^{2 i}-1\right)^{-1}} .
\end{aligned}
$$

Assume that the identities hold for $D_{2 k-1}$ and $D_{2 k}$. Since $D_{2 k+1}=u a^{2 k+1} D_{2 k}+D_{2 k-1}$,

$$
\begin{aligned}
D_{2 k+1} & \sum_{n=0}^{\infty}(u v)^{-n} a^{-n^{2}} \prod_{i=1}^{n}\left(a^{2 i}-1\right)^{-1} \\
= & \sum_{n=0}^{\infty}(u v)^{-n-k} a^{-n^{2}-(4 k+2) n-k(2 k+1)} \prod_{i=1}^{n}\left(a^{2 i}-1\right)^{-1} \\
& -\sum_{n=0}^{\infty}(u v)^{-n-k} a^{-n^{2}-4 k n-k(2 k+1)} \prod_{i=1}^{n}\left(a^{2 i}-1\right)^{-1} \\
= & \sum_{n=0}^{\infty}(u v)^{-n-k} a^{-n^{2}-(4 k+2) n-k(2 k+1)}\left(1-a^{2 n}\right) \prod_{i=1}^{n}\left(a^{2 i}-1\right)^{-1} \\
= & -\sum_{n=1}^{\infty}(u v)^{-n-k} a^{-n^{2}-(4 k+2) n-k(2 k+1)} \prod_{i=1}^{n-1}\left(a^{2 i}-1\right)^{-1} \\
= & -\sum_{n=0}^{\infty}(u v)^{-n-k-1} a^{-n^{2}-4(k+1) n-(k+1)(2 k+3)} \prod_{i=1}^{n}\left(a^{2 i}-1\right)^{-1} .
\end{aligned}
$$

Since $D_{2 k+2}=v a^{2 k+2} D_{2 k+1}+D_{2 k}$,

$$
\begin{aligned}
D_{2 k+2} & \sum_{n=0}^{\infty}(u v)^{-n} a^{-n^{2}} \prod_{i=1}^{n}\left(a^{2 i}-1\right)^{-1} \\
= & -\sum_{n=0}^{\infty} u^{-n-k-1} v^{-n-k} a^{-n^{2}-4(k+1) n-(k+1)(2 k+3)+2 k+2} \prod_{i=1}^{n}\left(a^{2 i}-1\right)^{-1} \\
& +\sum_{n=0}^{\infty} u^{-n-k-1} v^{-n-k} a^{-n^{2}-(4 k+2) n-(k+1)(2 k+1)} \prod_{i=1}^{n}\left(a^{2 i}-1\right)^{-1} \\
= & \sum_{n=0}^{\infty} u^{-n-k-1} v^{-n-k} a^{-n^{2}-4(k+1) n-(k+1)(2 k+1)}\left(-1+a^{2 n}\right) \prod_{i=1}^{n}\left(a^{2 i}-1\right)^{-1} \\
= & \sum_{n=0}^{\infty} u^{-n-k-2} v^{-n-k-1} a^{-(n+1)^{2}-4(k+1)(n+1)-(k+1)(2 k+1)} \prod_{i=1}^{n-1}\left(a^{2 i}-1\right)^{-1} \\
= & \sum_{n=0}^{\infty} u^{-n-k-2} v^{-n-k-1} a^{-n^{2}-(4 k+6) n-(k+2)(2 k+3)} \prod_{i=1}^{n}\left(a^{2 i}-1\right)^{-1} .
\end{aligned}
$$




\section{Diophantine approximations of Hurwitz continued fractions}

Let $p_{n} / q_{n}$ be the $n$th convergent of the tanh-type Hurwitz continued fraction [4, Theorem 5], [8, (1)]:

$$
\begin{aligned}
\alpha & =[0 ; u a, v(a+b), u(a+2 b), v(a+3 b), u(a+4 b), v(a+5 b), \ldots] \\
& =\frac{{ }_{0} F_{1}\left(; \frac{a}{b}+1 ; \frac{1}{u v b^{2}}\right)}{u a_{0} F_{1}\left(; \frac{a}{b} ; \frac{1}{u v b^{2}}\right)}=\frac{\sum_{n=0}^{\infty}(n !)^{-1} u^{-n-1}(v b)^{-n} \prod_{i=1}^{n}(a+b i)^{-1}}{\sum_{n=0}^{\infty}(n !)^{-1}(u v b)^{-n} \prod_{i=1}^{n-1}(a+b i)^{-1}},
\end{aligned}
$$

where

$$
{ }_{0} F_{1}(; c ; z)=\sum_{n=0}^{\infty} \frac{1}{(c)_{n}} \frac{z^{n}}{n !}
$$

is the confluent hypergeometric limit function. Here $(c)_{n}=c(c+1) \ldots(c+n-1)$ if $n \geq 1$ and $(c)_{0}=1$.

THeorem 14. For all $k \geq 1$,

$$
\begin{aligned}
D_{2 k-1} & =-\frac{{ }_{0} F_{1}\left(; \frac{a}{b}+2 k ; \frac{1}{u v b^{2}}\right)}{\left(u v b^{2}\right)^{k}\left(\frac{a}{b}\right)_{2 k 0} F_{1}\left(; \frac{a}{b} ; \frac{1}{u v b^{2}}\right)} \\
& =-\frac{\sum_{n=0}^{\infty}(n !)^{-1}(u v)^{-n-k} b^{-n} \prod_{i=1}^{n+2 k-1}(a+b i)^{-1}}{\sum_{n=0}^{\infty}(n !)^{-1}(u v b)^{-n} \prod_{i=1}^{n-1}(a+b i)^{-1}}, \\
D_{2 k} & =\frac{{ }_{0} F_{1}\left(; \frac{a}{b}+2 k+1 ; \frac{1}{u v b^{2}}\right)}{u a\left(u v b^{2}\right)^{k}\left(\frac{a}{b}+1\right)_{2 k 0} F_{1}\left(; \frac{a}{b} ; \frac{1}{u v b^{2}}\right)} \\
& =\frac{\sum_{n=0}^{\infty}(n !)^{-1} u^{-n-k-1} v^{-n-k} b^{-n} \prod_{i=1}^{n+2 k}(a+b i)^{-1}}{\sum_{n=0}^{\infty}(n !)^{-1}(u v b)^{-n} \prod_{i=1}^{n-1}(a+b i)^{-1}} .
\end{aligned}
$$

If we put $a=1$ and $b=2$ in Theorem 14 , we immediately get the following corollary.

COROLLARY 15. Let $p_{n} / q_{n}$ be the nth convergent of the continued fraction

$$
\sqrt{\frac{v}{u}} \tanh \frac{1}{\sqrt{u v}}=[0 ; \overline{(4 k-3) u,(4 k-1) v}]_{k=1}^{\infty} .
$$

Then for all $k \geq 1$,

$$
\begin{aligned}
D_{2 k-1} & =-\frac{{ }_{0} F_{1}\left(; \frac{1}{2}+2 k ; \frac{1}{4 u v}\right)}{(u v)^{k}(4 k-1) ! !_{0} F_{1}\left(; \frac{1}{2} ; \frac{1}{4 u v}\right)}, \\
D_{2 k} & =\frac{{ }_{0} F_{1}\left(; \frac{3}{2}+2 k ; \frac{1}{4 u v}\right)}{u^{k+1} v^{k}(4 k+1) ! !{ }_{0} F_{1}\left(; \frac{1}{2} ; \frac{1}{4 u v}\right)},
\end{aligned}
$$

where $(4 k-1) ! !=(4 k-1)(4 k-3) \cdots 3 \cdot 1$. 
Let $p_{n} / q_{n}$ be the $n$th convergent of the tan-type Hurwitz continued fraction [4, Theorem 6], [8, (2)]:

$$
\begin{aligned}
\alpha & =[0 ; u a-1,1, v(a+b)-2,1, u(a+2 b)-2,1, v(a+3 b)-2,1, \ldots] \\
& =\frac{{ }_{0} F_{1}\left(; \frac{a}{b}+1 ; \frac{-1}{u v b^{2}}\right)}{u a_{0} F_{1}\left(; \frac{a}{b} ; \frac{-1}{u v b^{2}}\right)}=\frac{\sum_{n=0}^{\infty}(-1)^{n}(n !)^{-1} u^{-n-1}(v b)^{-n} \prod_{i=1}^{n}(a+b i)^{-1}}{\sum_{n=0}^{\infty}(-1)^{n}(n !)^{-1}(u v b)^{-n} \prod_{i=1}^{n-1}(a+b i)^{-1}} .
\end{aligned}
$$

Theorem 16. For all $k \geq 1$,

$$
\begin{aligned}
D_{4 k-2} & =\frac{{ }_{0} F_{1}\left(; \frac{a}{b}+2 k ; \frac{-1}{u v b^{2}}\right)}{\left(u v b^{2}\right)^{k}\left(\frac{a}{b}\right)_{2 k} F_{1}\left(; \frac{a}{b} ; \frac{-1}{u v b^{2}}\right)} \\
& =\frac{\sum_{n=0}^{\infty}(-1)^{n}(n !)^{-1}(u v)^{-n-k} b^{-n} \prod_{i=1}^{n+2 k-1}(a+b i)^{-1}}{\sum_{n=0}^{\infty}(-1)^{n}(n !)^{-1}(u v b)^{-n} \prod_{i=1}^{n-1}(a+b i)^{-1}}, \\
D_{4 k} & =\frac{{ }_{0} F_{1}\left(; \frac{a}{b}+2 k+1 ; \frac{-1}{u v b^{2}}\right)}{u a\left(u v b^{2}\right)^{k}\left(\frac{a}{b}+1\right)_{2 k} F_{1}\left(; \frac{a}{b} ; \frac{-1}{u v b^{2}}\right)} \\
& =\frac{\sum_{n=0}^{\infty}(-1)^{n}(n !)^{-1} u^{-n-k-1} v^{-n-k} b^{-n} \prod_{i=1}^{n+2 k}(a+b i)^{-1}}{\sum_{n=0}^{\infty}(-1)^{n}(n !)^{-1}(u v b)^{-n} \prod_{i=1}^{n-1}(a+b i)^{-1}} .
\end{aligned}
$$

Remark 17. For odd $k$, the recurrence relation $D_{k}=D_{k+1}-D_{k-1}$ gives a formula for $D_{k}$.

If we put $a=1$ and $b=2$ in Theorem 16 , we immediately get the following corollary.

Corollary 18. Let $p_{n} / q_{n}$ be the nth convergent of the continued fraction

$$
\sqrt{\frac{v}{u}} \tan \frac{1}{\sqrt{u v}}=[0 ; u-1, \overline{1,(4 k-3) v-2,1,(4 k+1) u-2,1}]_{k=1}^{\infty}
$$

Then for all $k \geq 1$,

$$
\begin{aligned}
& D_{4 k-2}=\frac{{ }_{0} F_{1}\left(; \frac{1}{2}+2 k ; \frac{-1}{4 u v}\right)}{(u v)^{k}(4 k-1) ! !{ }_{0} F_{1}\left(; \frac{1}{2} ; \frac{-1}{4 u v}\right)}, \\
& D_{4 k}=\frac{{ }_{0} F_{1}\left(; \frac{3}{2}+2 k ; \frac{-1}{4 u v}\right)}{u^{k+1} v^{k}(4 k+1) ! !{ }_{0} F_{1}\left(; \frac{1}{2} ; \frac{-1}{4 u v}\right)},
\end{aligned}
$$

where $(4 k-1) ! !=(4 k-1)(4 k-3) \cdots 3 \cdot 1$. 
Let $p_{n} / q_{n}$ be the $n$th convergent of the $e$-type Hurwitz continued fraction (see [6, Theorem 1], [8, (3)]):

$$
\begin{aligned}
\alpha & =[0 ; \overline{u(a+b k)-1,1, v-1}]_{k=1}^{\infty} \\
& =\frac{v_{0} F_{1}\left(; \frac{a}{b}+2 ; \frac{1}{u^{2} v^{2} b^{2}}\right)}{u v(a+b)_{0} F_{1}\left(; \frac{a}{b}+1 ; \frac{1}{u^{2} v^{2} b^{2}}\right)-{ }_{0} F_{1}\left(; \frac{a}{b}+2 ; \frac{1}{u^{2} v^{2} b^{2}}\right)} \\
& =\frac{\sum_{n=0}^{\infty} u^{-2 n-1} v^{-2 n} b^{-n}(n !)^{-1} \prod_{i=1}^{n+1}(a+b i)^{-1}}{\sum_{n=0}^{\infty} b^{-n}(n !)^{-1}\left((u v)^{-2 n} \prod_{i=1}^{n}(a+b i)^{-1}-(u v)^{-2 n-1} \prod_{i=1}^{n+1}(a+b i)^{-1}\right)}
\end{aligned}
$$

THEOREM 19. For all $k \geq 1$,

$$
\begin{aligned}
D_{3 k-1} & =\frac{(-1)^{k}}{(u v b)^{k}\left(\frac{a}{b}+2\right)_{k}} \frac{{ }_{0} F_{1}\left(; \frac{a}{b}+k+2 ; \frac{1}{u^{2} v^{2} b^{2}}\right)-u v(a+b(k+1))_{0} F_{1}\left(; \frac{a}{b}+k+1 ; \frac{1}{u^{2} v^{2} b^{2}}\right)}{u v(a+b){ }_{0} F_{1}\left(; \frac{a}{b}+1 ; \frac{1}{u^{2} v^{2} b^{2}}\right)-{ }_{0} F_{1}\left(; \frac{a}{b}+2 ; \frac{1}{u^{2} v^{2} b^{2}}\right)} \\
& =\frac{(-1)^{k} \sum_{n=0}^{\infty} b^{-n}(n !)^{-1}\left((u v)^{-2 n-k-1} \prod_{i=1}^{n+k+1}(a+b i)^{-1}-(u v)^{-2 n-k} \prod_{i=1}^{n+k}(a+b i)^{-1}\right)}{\sum_{n=0}^{\infty} b^{-n}(n !)^{-1}\left((u v)^{-2 n} \prod_{i=1}^{n}(a+b i)^{-1}-(u v)^{-2 n-1} \prod_{i=1}^{n+1}(a+b i)^{-1}\right)} \\
D_{3 k} & =\frac{(-1)^{k} v}{(u v b)^{k}\left(\frac{a}{b}+2\right)_{k}} \frac{{ }_{0} F_{1}\left(; \frac{a}{b}+k+2 ; \frac{1}{u^{2} v^{2} b^{2}}\right)}{u v(a+b){ }_{0} F_{1}\left(; \frac{a}{b}+1 ; \frac{1}{u^{2} v^{2} b^{2}}\right)-{ }_{0} F_{1}\left(; \frac{a}{b}+2 ; \frac{1}{u^{2} v^{2} b^{2}}\right)} \\
& =\frac{(-1)^{k} \sum_{n=0}^{\infty} u^{-2 n-k-1} v^{-2 n-k} b^{-n}(n !)^{-1} \prod_{i=1}^{n+k+1}(a+b i)^{-1}}{\sum_{n=0}^{\infty} b^{-n}(n !)^{-1}\left((u v)^{-2 n} \prod_{i=1}^{n}(a+b i)^{-1}-(u v)^{-2 n-1} \prod_{i=1}^{n+1}(a+b i)^{-1}\right)}
\end{aligned}
$$

REMARK 20. The recurrence relation $D_{3 k-2}=D_{3 k-1}-D_{3 k-3}$ gives a formula for $D_{3 k-2}$.

Corollary 21. Let $p_{n} / q_{n}$ be the nth convergent of the continued fraction

$$
e^{1 / s}-1=[0 ; \overline{(2 k-1) s-1,1,1}]_{k=1}^{\infty} .
$$

Then for all $k \geq 1$,

$$
\begin{gathered}
D_{3 k-1}=\frac{(-1)^{k} e^{1 /(2 s)}}{s^{k}} \sum_{n=0}^{\infty} \frac{(n+k) !}{n !}\left(\frac{1}{(2 s)^{2 n+1}(2 n+2 k+1) !}-\frac{1}{(2 s)^{2 n}(2 n+2 k) !}\right), \\
D_{3 k}=\frac{(-1)^{k} e^{1 /(2 s)}}{s^{k+1}} \sum_{n=0}^{\infty} \frac{(n+k) !}{(2 s)^{2 n} n !(2 n+2 k+1) !} .
\end{gathered}
$$

Let $p_{n} / q_{n}$ be the $n$th convergent of another $e$-type Hurwitz continued fraction (see [6, Theorem 2], [8, (4)]):

$$
\begin{aligned}
\alpha & =[0 ; \overline{v-1,1, u(a+b k)-1}]_{k=1}^{\infty} \\
& =\frac{{ }_{0} F_{1}\left(; \frac{a}{b}+2 ; \frac{1}{u^{2} v^{2} b^{2}}\right)}{u v^{2}(a+b)_{0} F_{1}\left(; \frac{a}{b}+1 ; \frac{1}{u^{2} v^{2} b^{2}}\right)}+\frac{1}{v} .
\end{aligned}
$$


Theorem 22. For all $k \geq 1$,

$$
\begin{gathered}
D_{3 k-1}=\frac{(-1)^{k-1} \sum_{n=0}^{\infty}(u v)^{-2 n-k} b^{-n}(n !)^{-1} \prod_{i=1}^{n+k}(a+b i)^{-1}}{\sum_{n=0}^{\infty}(u v)^{-2 n} b^{-n}(n !)^{-1} \prod_{i=1}^{n}(a+b i)^{-1}}, \\
D_{3 k}=\frac{(-1)^{k} \sum_{n=0}^{\infty} b^{-n}(n !)^{-1}\left(u^{-2 n-k} v^{-2 n-k-1} \prod_{i=1}^{n+k}(a+b i)^{-1}+u^{-2 n-k-1} v^{-2 n-k-2} \prod_{i=1}^{n+k+1}(a+b i)^{-1}\right)}{\sum_{n=0}^{\infty}(u v)^{-2 n} b^{-n}(n !)^{-1} \prod_{i=1}^{n}(a+b i)^{-1}} .
\end{gathered}
$$

REMARK 23. The recurrence relation $D_{3 k-2}=D_{3 k-1}-D_{3 k-3}$ gives a formula for $D_{3 k-2}$.

\section{Proof of Theorem 14 and Corollaries 21 and 26}

We shall prove Theorem 14 by induction. Other theorems can be proven similarly. Let

$$
\begin{aligned}
\alpha & =[0 ; u a, v(a+b), u(a+2 b), v(a+3 b), u(a+4 b), v(a+5 b), \ldots] \\
& =\frac{\sum_{n=0}^{\infty}(n !)^{-1} u^{-n-1}(v b)^{-n} \prod_{i=1}^{n}(a+b i)^{-1}}{\sum_{n=0}^{\infty}(n !)^{-1}(u v b)^{-n} \prod_{i=1}^{n-1}(a+b i)^{-1}} \\
& =\frac{\sum_{n=0}^{\infty}(n !)^{-1} u^{-n-1}(v b)^{-n} \prod_{i=1}^{n}(a+b i)^{-1}}{\sum_{n=0}^{\infty}(n !)^{-1}(u v b)^{-n} \prod_{i=1}^{n-1}(a+b i)^{-1}} .
\end{aligned}
$$

The identities hold for $D_{0}$ and $D_{1}$ because

$$
\begin{aligned}
D_{0} & =q_{0} \alpha-p_{0}=\alpha \\
& =\frac{\sum_{n=0}^{\infty}(n !)^{-1} u^{-n-1}(v b)^{-n} \prod_{i=1}^{n}(a+b i)^{-1}}{\sum_{n=0}^{\infty}(n !)^{-1}(u v b)^{-n} \prod_{i=1}^{n-1}(a+b i)^{-1}}
\end{aligned}
$$

and

$$
\begin{aligned}
D_{1} & =q_{1} \alpha-p_{1}=u a \alpha-1 \\
& =\frac{\left(u a \sum_{n=0}^{\infty}(n !)^{-1} u^{-n-1}(v b)^{-n}-\sum_{n=0}^{\infty}(n !)^{-1}(u v b)^{-n}(a+b n)\right) \prod_{i=1}^{n-1}(a+b i)^{-1}}{\sum_{n=0}^{\infty}(n !)^{-1}(u v b)^{-n} \prod_{i=1}^{n-1}(a+b i)^{-1}} \\
& =-\frac{\sum_{n=1}^{\infty}((n-1) !)^{-1}(u v)^{-n} b^{-n+1} \prod_{i=1}^{n}(a+b i)^{-1}}{\sum_{n=0}^{\infty}(n !)^{-1}(u v b)^{-n} \prod_{i=1}^{n-1}(a+b i)^{-1}} \\
& =-\frac{\sum_{n=0}^{\infty}(n !)^{-1}(u v)^{-n-1} b^{-n} \prod_{i=1}^{n+1}(a+b i)^{-1}}{\sum_{n=0}^{\infty}(n !)^{-1}(u v b)^{-n} \prod_{i=1}^{n-1}(a+b i)^{-1}} .
\end{aligned}
$$

Next, we shall prove Corollary 21. Put $a=-1, b=2, u=s$ and $v=2$ in Theorem 19. Since

$$
\prod_{i=1}^{n}(a+b i)^{-1}=\prod_{i=1}^{n}(2 i-1)^{-1}=\frac{2^{n} n !}{(2 n) !},
$$


we obtain

$$
\begin{aligned}
& \sum_{n=0}^{\infty} b^{-n}(n !)^{-1}\left((u v)^{-2 n} \prod_{i=1}^{n}(a+b i)^{-1}-(u v)^{-2 n-1} \prod_{i=1}^{n+1}(a+b i)^{-1}\right) \\
& =\sum_{n=0}^{\infty} \frac{1}{(2 s)^{2 n}(2 n) !}-\sum_{n=0}^{\infty} \frac{1}{(2 s)^{2 n+1}(2 n+1) !} \\
& =\cosh \left(\frac{1}{2 s}\right)-\sinh \left(\frac{1}{2 s}\right)=e^{-1 /(2 s)} .
\end{aligned}
$$

On the other hand, the numerator of $D_{3 k-1}$ is equal to

$$
\frac{(-1)^{k}}{s^{k}} \sum_{n=0}^{\infty} \frac{(n+k) !}{n !}\left(\frac{1}{(2 s)^{2 n+1}(2 n+2 k+1) !}-\frac{1}{(2 s)^{2 n}(2 n+2 k) !}\right) .
$$

The numerator of $D_{3 k}$ is equal to

$$
\frac{(-1)^{k}}{s^{k+1}} \sum_{n=0}^{\infty} \frac{(n+k) !}{(2 s)^{2 n} n !(2 n+2 k+1) !} .
$$

\section{Diophantine approximations in terms of integrals}

In [12, Theorem 1], for the continued fraction

$$
\sqrt{\frac{v}{u}} \tanh \frac{1}{\sqrt{u v}}=[0 ; \overline{(4 k-3) u,(4 k-1) v}]_{k=1}^{\infty},
$$

we have

$$
\begin{aligned}
D_{2 k-1} & =\frac{1}{e^{2 / \sqrt{u v}}+1}\left(\frac{4}{u v}\right)^{k} \int_{0}^{1} \frac{x^{2 k-1}(x-1)^{2 k-1}}{(2 k-1) !} e^{2 x / \sqrt{u v}} d x, \\
D_{2 k} & =\frac{2}{\left(e^{2 / \sqrt{u v}}+1\right) u}\left(\frac{4}{u v}\right)^{k} \int_{0}^{1} \frac{x^{2 k}(x-1)^{2 k}}{(2 k) !} e^{2 x / \sqrt{u v}} d x .
\end{aligned}
$$

Together with the identities in Corollary 15 we get the following corollary.

Corollary 24.

$$
\begin{aligned}
& \left(e^{2 / \sqrt{u v}}+1\right) \frac{{ }_{0} F_{1}\left(; \frac{1}{2}+2 k ; \frac{1}{4 u v}\right)}{{ }_{0} F_{1}\left(; \frac{1}{2} ; \frac{1}{4 u v}\right)} \\
& =-2^{2 k}(4 k-1) ! ! \int_{0}^{1} \frac{x^{2 k-1}(x-1)^{2 k-1}}{(2 k-1) !} e^{2 x / \sqrt{u v}} d x \\
& \left(e^{2 / \sqrt{u v}}+1\right) \frac{{ }_{0} F_{1}\left(; \frac{3}{2}+2 k ; \frac{1}{4 u v}\right)}{{ }_{0} F_{1}\left(; \frac{1}{2} ; \frac{1}{4 u v}\right)} \\
& =2^{2 k+1}(4 k+1) ! ! \int_{0}^{1} \frac{x^{2 k}(x-1)^{2 k}}{(2 k) !} e^{2 x / \sqrt{u v}} d x .
\end{aligned}
$$


In [12, Theorem 2], for the continued fraction

$$
\sqrt{\frac{v}{u}} \tan \frac{1}{\sqrt{u v}}=[0 ; u-1, \overline{1,(4 k-3) v-2,1,(4 k+1) u-2,1}]_{k=1}^{\infty},
$$

we have

$$
\begin{aligned}
D_{4 k-2} & =-\frac{1}{e^{2 \sqrt{-1} / \sqrt{u v}}+1}\left(\frac{4}{u v}\right)^{k} \int_{0}^{1} \frac{x^{2 k-1}(x-1)^{2 k-1}}{(2 k-1) !} e^{2 \sqrt{-1} x / \sqrt{u v}} d x, \\
D_{4 k} & =\frac{2}{\left(e^{2 \sqrt{-1} / \sqrt{u v}}+1\right) u}\left(\frac{4}{u v}\right)^{k} \int_{0}^{1} \frac{x^{2 k}(x-1)^{2 k}}{(2 k) !} e^{2 \sqrt{-1} x / \sqrt{u v}} d x .
\end{aligned}
$$

Together with the identities in Corollary 18 we get the following corollary.

Corollary 25.

$$
\begin{aligned}
& \left(e^{2 \sqrt{-1} / \sqrt{u v}}+1\right) \frac{{ }_{0} F_{1}\left(; \frac{1}{2}+2 k ; \frac{-1}{4 u v}\right)}{{ }_{0} F_{1}\left(; \frac{1}{2} ; \frac{-1}{4 u v}\right)} \\
& =-2^{2 k}(4 k-1) ! ! \int_{0}^{1} \frac{x^{2 k-1}(x-1)^{2 k-1}}{(2 k-1) !} e^{2 \sqrt{-1} x / \sqrt{u v}} d x, \\
& \left(e^{2 \sqrt{-1} / \sqrt{u v}}+1\right) \frac{{ }_{0} F_{1}\left(; \frac{3}{2}+2 k ; \frac{-1}{4 u v}\right)}{{ }_{0} F_{1}\left(; \frac{1}{2} ; \frac{-1}{4 u v}\right)} \\
& =2^{2 k+1}(4 k+1) ! ! \int_{0}^{1} \frac{x^{2 k}(x-1)^{2 k}}{(2 k) !} e^{2 \sqrt{-1} x / \sqrt{u v}} d x .
\end{aligned}
$$

In $[11,15]$, for the continued fraction

$$
e^{1 / s}=[1 ; \overline{(2 k-1) s-1,1,1}]_{k=1}^{\infty},
$$

we have

$$
\begin{aligned}
D_{3 k-1} & =-\frac{1}{s^{k}} \int_{0}^{1} \frac{x^{k-1}(x-1)^{k}}{(k-1) !} e^{x / s} d x, \\
D_{3 k} & =\frac{1}{s^{k+1}} \int_{0}^{1} \frac{x^{k}(x-1)^{k}}{k !} e^{x / s} d x .
\end{aligned}
$$

Together with the identities in Corollary 21 we get the following corollary.

Corollary 26.

$$
\begin{aligned}
e^{1 /(2 s)} & \sum_{n=0}^{\infty} \frac{(n+k) !}{n !}\left(\frac{1}{(2 s)^{2 n+1}(2 n+2 k+1) !}-\frac{1}{(2 s)^{2 n}(2 n+2 k) !}\right) \\
= & (-1)^{k+1} \int_{0}^{1} \frac{x^{k-1}(x-1)^{k}}{(k-1) !} e^{x / s} d x, \\
e^{1 /(2 s)} & \sum_{n=0}^{\infty} \frac{(n+k) !}{(2 s)^{2 n} n !(2 n+2 k+1) !}=(-1)^{k} \int_{0}^{1} \frac{x^{k}(x-1)^{k}}{k !} e^{x / s} d x .
\end{aligned}
$$




\section{Some additional comments}

The identities in Theorems 1 to 10 may be obtained in indirect ways. For example, let $p_{n} / q_{n}$ be the $n$th convergent of the Tasoev continued fraction $\alpha=\left[0 ; \overline{u a^{2 k-1}, v a^{2 k}}\right]_{k=1}^{\infty}$ in Theorem 1. Then we know that for all $n \geq 1$,

$$
\begin{gathered}
p_{2 n-1}=\sum_{v=0}^{n-1}(u v)^{v} a^{v(2 v+3)} \prod_{i=1}^{n-v-1} \frac{a^{2(2 v+i)}-1}{a^{2 i}-1}, \\
p_{2 n}=\sum_{v=0}^{n-1} u^{v} v^{v+1} a^{(v+2)(2 v+1)} \prod_{i=1}^{n-v-1} \frac{a^{2(2 v+i+1)}-1}{a^{2 i}-1}, \\
q_{2 n-1}=\sum_{v=0}^{n-1} u^{v+1} v^{v} a^{(v+1)(2 v+1)} \prod_{i=1}^{n-v-1} \frac{a^{2(2 v+i+1)}-1}{a^{2 i}-1}, \\
q_{2 n}=\sum_{v=0}^{n}(u v)^{v} a^{v(2 v+1)} \prod_{i=1}^{n-v} \frac{a^{2(2 v+i)}-1}{a^{2 i}-1} .
\end{gathered}
$$

(These identities are also proven by induction.) After some manipulations of series,

$$
\begin{aligned}
& \left(q_{2 k-1} \alpha-p_{2 k-1}\right) \sum_{n=0}^{\infty}(u v)^{-n} a^{-n^{2}} \prod_{i=1}^{n}\left(a^{2 i}-1\right)^{-1} \\
& =-\sum_{n=0}^{\infty}(u v)^{-n-k} a^{-n^{2}-4 k n-k(2 k+1)} \prod_{i=1}^{n}\left(a^{2 i}-1\right)^{-1}
\end{aligned}
$$

and

$$
\begin{aligned}
\left(q_{2 k} \alpha\right. & \left.-p_{2 k}\right) \sum_{n=0}^{\infty}(u v)^{-n} a^{-n^{2}} \prod_{i=1}^{n}\left(a^{2 i}-1\right)^{-1} \\
& =\sum_{n=0}^{\infty} u^{-n-k-1} v^{-n-k} a^{-n^{2}-(4 k+2) n-(k+1)(2 k+1)} \prod_{i=1}^{n}\left(a^{2 i}-1\right)^{-1} .
\end{aligned}
$$

But these manipulations are not easy.

\section{Acknowledgement}

The author thanks the anonymous referee for some valuable suggestions.

\section{References}

[1] E. B. Burger, Exploring the Number Jungle: A Journey into Diophantine Analysis, Student Mathematical Library, 8 (American Mathematical Society, Providence RI, 2000).

[2] H. Cohn, 'A short proof of the simple continued fraction expansion of e', Amer. Math. Monthly 113 (2006), 57-62.

[3] T. Komatsu, 'On Tasoev's continued fractions', Math. Proc. Cambridge Philos. Soc. 134 (2003), $1-12$. 
[4] T. Komatsu, 'On Hurwitzian and Tasoev's continued fractions', Acta Arith. 107 (2003), 161-177.

[5] T. Komatsu, 'Tasoev's continued fractions and Rogers-Ramanujan continued fractions', J. Number Theory 109 (2004), 27-40.

[6] T. Komatsu, 'Hurwitz and Tasoev continued fractions', Monatsh. Math. 145 (2005), 47-60.

[7] T. Komatsu, 'An algorithm of infinite sums representations and Tasoev continued fractions', Math. Comp. 74 (2005), 2081-2094.

[8] T. Komatsu, 'Hurwitz continued fractions with confluent hypergeometric functions', Czechoslovak Math. J. 57 (2007), 919-932.

[9] T. Komatsu, 'Tasoev continued fractions with long period', Far East J. Math. Sci. (FJMS) 28 (2008), 89-121.

[10] T. Komatsu, 'More on Hurwitz and Tasoev continued fractions', Sarajevo J. Math. 4 (2008), $155-180$.

[11] T. Komatsu, 'A diophantine approximation of $e^{1 / s}$ in terms of integrals', Tokyo J. Math. 32 (2009), 159-176.

[12] T. Komatsu, 'Diophantine approximations of tanh, tan, and linear forms of $e$ in terms of integrals', Rev. Roumaine Math. Pures Appl. 54 (2009), 223-242.

[13] T. Matala-Aho and V. Merilä, 'On Diophantine approximations of Ramanujan type $q$-continued fractions', J. Number Theory 129 (2009), 1044-1055.

[14] J. Mc Laughlin, 'Some new families of Tasoevian and Hurwitzian continued fractions', Acta Arith. 135 (2008), 247-268.

[15] T. Osler, 'A proof of the continued fraction expansion of $e^{1 / M}$, Amer. Math. Monthly 113 (2006), 62-66.

[16] O. Perron, Die Lehre von den Kettenbrüchen, Band I (Teubner, Stuttgart, 1954).

[17] B. G. Tasoev, 'Rational approximations to certain numbers', Mat. Zametki 67 (2000), 931-937 (Russian), English transl. in Math. Notes 67 (2000), 786-791.

TAKAO KOMATSU, Graduate School of Science and Technology, Hirosaki University, Hirosaki 036-8561, Japan

e-mail: komatsu@cc.hirosaki-u.ac.jp 